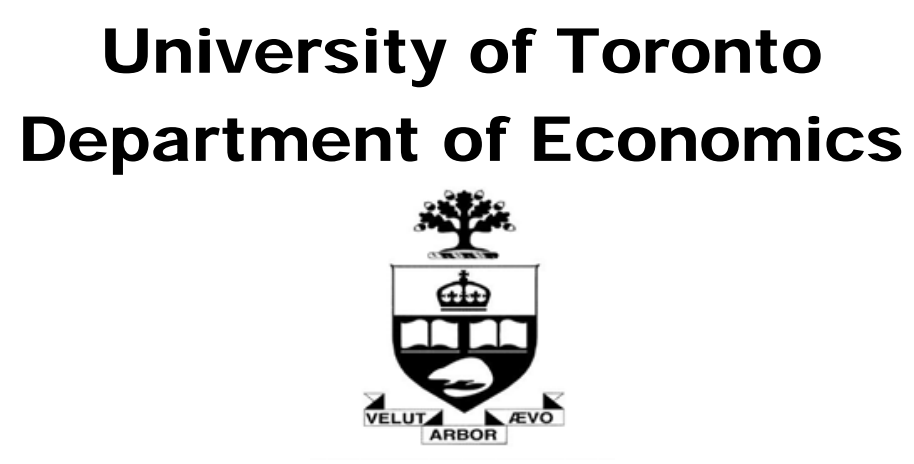

Working Paper 386

Welfare Rankings From Multivariate Data, A Non-Parametric Approach

By Gordon Anderson, lan Crawford and Andrew Leicester

January 14, 2010 


\title{
Welfare rankings from multivariate data, a nonparametric approach
}

\author{
Gordon Anderson*, Ian Crawford† Andrew Leicester ${ }^{\ddagger}$
}

January 12, 2010

\begin{abstract}
Economic and social welfare is inherently multidimensional. However, choosing a measure which combines several indicators is difficult and may have unintendend and undesirable effects on the incentives of policy makers. We develop a nonparametric empirical method for deriving welfare rankings based on data envelopment, which avoids the need to specify a weighting scheme. The results are valid for all possible social welfare functions which share certain canonical properties. We apply this method to data on Human Development.
\end{abstract}

Acknowledgments. We would like to thank Ian Preston and Martin Ravallion for helpful discussions and comments. Financial support from the ESRC grant number RES000-22-0393 is gratefully acknowledged. The authors are responsible for all errors.

\section{Introduction}

Sen and many others have consistently and persuasively argued that aspects of well-being, be it inequality, deprivation or polarization, are intrinsically many-dimensioned things (for example Sen (1995), Anand and Sen (1997), Atkinson (2003), Bourguignon and Chakravarty (2003), Kolm (1977), Maasoumi (1986) and the essays in Grusky and Kanbur (2006)). An individual's functionings and capabilities are bounded by many sensibilities, the extent of their freedoms, limitations afforded by their health, knowledge and skill set and ultimately their capacity to buy goods and leisure. Evaluation of these various aspects of societal wellbeing demands recognition of its multi-dimensional nature.

Whilst the argument that well-being is multi-dimensional is well taken it is often still extremely useful to be able to order and to compare states characterized in many dimensions. Policy makers, for example, frequently require some means of comparison that is complete.

\footnotetext{
*University of Toronto

${ }^{\dagger}$ University of Oxford and IFS.

${ }^{\ddagger}$ Institute for Fiscal Studies
} 
Thus beyond the difficulties surrounding measurement of these many sensibilities, an evaluation of overall well-being calls for some means of aggregating across them. Therein lies the difficulty, for while there may be general agreement on an aggregation method, the specific weights to be attached to each sensibility are a matter of some dispute. The choice of any particular weighting scheme is somewhat arbitrary, and unfortunately once made it rules out other equally plausible but no less arbitrary weighting schemes.

A good example of this problem is the United Nations Human Development Index (HDI) which aims to provide a single summary measure of the relative development status of different countries. Based upon indices of three dimensions, education (a combination of literacy and school enrolment rates), life expectancy and GDP per capita, it simply adds the three indices up and divides by three, attaching equal weight to each sensibility. The implication is a one percent increase in any one of the factors will have an effect on 'development' identical to that of a corresponding change in any other, and this will be the case whatever the levels of the individual factors. This has obvious implications for policy design, since a policy maker's attention will be directed to those factors which have the greatest weight in the aggregation scheme. Whether or not this is desirable should be a matter of conscious and careful consideration, rather than as the unintended consequence of the choice of a mathematical function.

This paper offers a constructive approach to the aggregation problem. We consider the situation in which we have data recording various aspects of well-being for a cross section of observations (life-expectancy, income and education, for example, for a cross section of countries as is the case for the UN HDI data). We show how two-sided bounds can be placed on a welfare index for each observation using only the assumptions that well-being is non-decreasing and weakly quasi-concave with respect to these indicators. Our approach is applied directly to the data and is fully nonparametric in the sense that it does not require us to make any further assumptions on the functional form of the welfare function, nor does it require us to estimate any functions of the data. Indeed the method we are suggesting can be applied to very small datasets (as well as to large ones) where statistical techniques - and especially nonparametric statistical techniques - could not be relied upon. A useful feature of our approach is that, since it is nonparametric and nonstochastic, the methodology is easily replicable requiring nothing more complex than standard linear programming techniques. We illustrate the method using the most recent UN HDI data. We show that it is indeed possible to recover informative two-sided bounds on the welfare index. Because the bounds encompass the entire set of welfare indices consistent with monotonicity and quasi-concavity, these bounds can be used as a computationally convenient robustness check on parametric methods. In 
other words researchers do not have to go through the unending tasking of computing all of the alternative measures, but instead simply have to compute the bounds. The approach set out in this paper also suggests a potential research program which might extend the work described in a number of ways.

The plan of the paper is as follows. Section 2 sets out the basic theory relating to our approach, describes the calculation of the bounds and provides two key propositions concerning them. Section 3 provides an empirical illustration which uses the UN HDI data and describes our experience with applying the methodology. Section 4 concludes and considers the shape of future work in this area.

\section{Theory}

\subsection{The distance function}

Suppose that there are $m$ variables recording different aspects of social and economic welfare for each of $n$ observations in a dataset (this dataset may be composed of individuals, communities or countries and is indexed $i=1, \ldots, n)$. In what follows we assume either that these variables are non-negative, or are transformed to be such. Let $\mathbf{x}_{i} \in \mathbb{R}_{+}^{m}$ denote the $i$ 'th observation. Let $\mathbf{X}$ be the $(m \times n)$ matrix of all of the $n$ observations. Let $W: \mathbb{R}_{+}^{m} \rightarrow \mathbb{R}$ denote a function which aggregates the variables associated with an observation into a single scalar measure. We can think of $W$ as representing a welfare/well-being function so that $W\left(\mathbf{x}_{i}\right)$ measures the welfare of $i$ 'th observation. We will make the following two assumptions regarding the welfare function.

A1. Monotonicity: $W(\mathbf{x}) \geq W(\mathbf{y})$ if $\mathbf{x} \geq \mathbf{y}$.

A2. Quasi-concavity: $W(\mathbf{x})=W(\mathbf{y}) \leq W(\alpha \mathbf{x}+(1-\alpha) \mathbf{y}) \quad \forall \alpha \in[0,1]$

Monotonicity means that the well-being does not fall with an increase in the measured variables. Quasi-concavity means that for a given distribution of $\mathbf{x}$ welfare is (weakly) increased by any inequality reducing reallocation between observations.

In this paper we focus, not on the primal welfare function, but on a dual representation of it called the distance function ${ }^{1}$. The distance function measures the amount by which one has to scale the variable vector of an observation so that it achieves some reference welfare

\footnotetext{
${ }^{1}$ See, for example, Deaton (1979) and Deaton and Muellbauer (1980). The term is from the economics literature (Shephard (1953) for example). In the mathematics literature the same object is known as a gauge function (see Rockafellar (1970), for example.)
} 
level. It is defined as follows

$$
d(\mathbf{x}, W)=\min _{d \geq 0}\{d: W(d \mathbf{x}) \geq W\}
$$

The distance function is decreasing in $\mathbf{x}$, increasing in $W$ and homogeneous of degree one in $\mathbf{x}$. The distance index can thought of as a (Malmquist) quantity index number measuring the "size" of $\mathbf{x}$ relative to the reference welfare level $W^{2}$. To illustrate consider Figure 1 which shows the general idea behind this index. There are two variables $\left\{x^{1}, x^{2}\right\}$, one measured on each axis and a single observation $\left(\mathbf{x}_{i}\right)$. The curve $W$ represents all of the combinations of the two variables which can produce a reference level of welfare. This curve is downward-sloping and convex to the origin thanks to the two assumptions above. The value of the distance function is given by the scalar value $d_{i}$. This is the smallest number by which $\mathbf{x}_{i}$ can be scaled such that the bundle $d_{i} \mathbf{x}_{i}$ lies on or above $W$. In this case $d_{i} \approx \frac{1}{2}$ which means that an equi-proportional reduction of about $50 \%$ in all of the variables would place the observation at the required reference welfare. Lower (respectively higher) values of $d_{i}$ indicate higher (lower) welfare compared to $W$. That distance functions in general depend on the location of $\mathbf{x}_{i}$ the welfare function and the reference welfare level is clearly illustrated by the figure by considering how the construction would vary with these factors. Another feature which is implicit in the figure is that knowing the distance function is as good as knowing the welfare function itself (you can identify the curve by knowing the value of $d_{i}$ for all possible locations of $\mathbf{x}_{i}$ and connecting up the set of points such that $d_{i}=1$ ).

FiguRE 1: The distance function

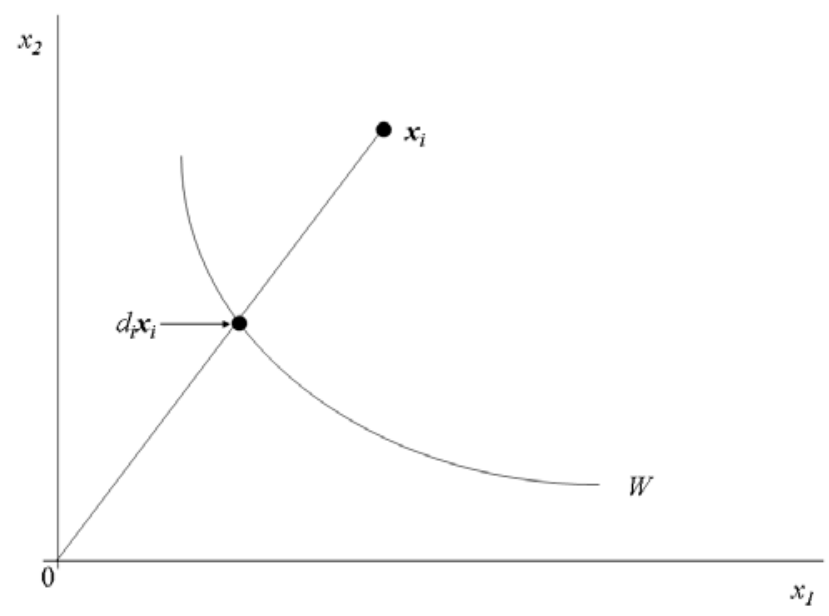

\footnotetext{
${ }^{2}$ This is a standard method in the index number literature. See Malmquist (1953).
} 
Since the distance function is a dual representation of the welfare function we could choose a formula for either and proceed to apply them a dataset in order to investigate welfare rankings. However, given the forgoing discussion about the difficulties involved in agreeing on a specific welfare aggregator, the challenge is to try to develop methods which are nonparametric; that is, which do not depend upon the functional form of a specific aggregator. In the next section we show that it is possible to recover bounds on the distance function which are valid for all possible choices of aggregator which satisfy monotonicity and quasi-concavity given an appropriate choice of the reference observation.

\subsection{Bounding the Distance Function}

Consider the following reference welfare level

$$
W^{*}=\min _{j}\left\{W\left(\mathbf{x}_{j}\right): \mathbf{x}_{j} \in \mathbf{X}, W \text { satisfies A1 and A2 }\right\}
$$

That is, the reference welfare level is the welfare associated the worst off observation where the welfare measure is required to satisfy monotonicity and quasi-concavity. Given this reference welfare curve it is possible to recover two-sided bounds on the distance index for each observation in the data without making further parametric assumptions about the welfare function. The formal result is stated next.

Proposition 1. $d\left(\mathbf{x}_{i}, W^{*}\right) \in\left[\underline{D_{i}}, \overline{D_{i}}\right]$ for $W^{*}=\min _{j}\left\{W\left(\mathbf{x}_{j}\right): \mathbf{x}_{j} \in \mathbf{X}, W\right.$ satisfies $A .1$ and A.2\} where

$$
\begin{aligned}
& \underline{D}_{i}=\min _{m}\left\{\min _{j}\left\{\frac{x_{j}^{m}}{x_{i}^{m}}\right\} \text { for } j=1, \ldots, n\right\} \\
& \overline{D_{i}}=\underset{\boldsymbol{\lambda} \geq \mathbf{0}_{n}, \overline{D_{i}} \geq 0}{\arg \min }\left[\mathbf{0}_{n}^{\prime}, 1\right]\left[\frac{\boldsymbol{\lambda}}{\overline{D_{i}}}\right] \text { such that }\left[\begin{array}{cc}
\mathbf{X} & -\mathbf{x}_{i} \\
\mathbf{1}_{n}^{\prime} & 0
\end{array}\right]\left[\begin{array}{c}
\boldsymbol{\lambda} \\
\overline{D_{i}}
\end{array}\right] \stackrel{\leq}{=}\left[\begin{array}{c}
\mathbf{0}_{n} \\
1
\end{array}\right]
\end{aligned}
$$

Proof. See Appendix.

The proof of the proposition can be found in the Appendix. However the general intuition for the result can be seen graphically Figure 2. Figure 2a illustrates a situation in which we have three observations $\left\{\mathbf{x}_{i}, \mathbf{x}_{j}, \mathbf{x}_{k}\right\}$. 
Figure 2A: Two -sided bounds on the distance function

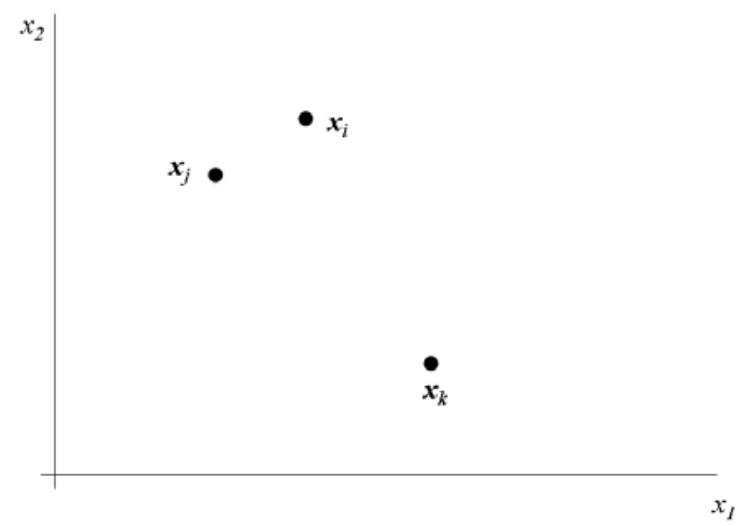

Our aim is to place bounds on the distance function referenced on the worst-off observation. The first question therefore is: which is the reference observation? Clearly (by monotonicity) it cannot be observation $i$. But it can, given an appropriate choice of $W$, be either $j$ or $k$ so we will have to consider both options. Begin with observation $j$. The downward-sloping dashed curve in the left hand panel of Figure 2B illustrates a potential reference welfare curve with the required properties such that $j$ is the worst off observation. Any such curve is admissible as a reference curve as long as it remains between the two shaded areas. If it crossed these bounds it would violate either monotonicity or quasiconcavity (or both). The right hand panel in Figure 2B shows a similar bound on the welfare curve through observation $k$ in the case that observation $k$ is the worst off.

Figure 2B: Two -sided bounds on the distance function
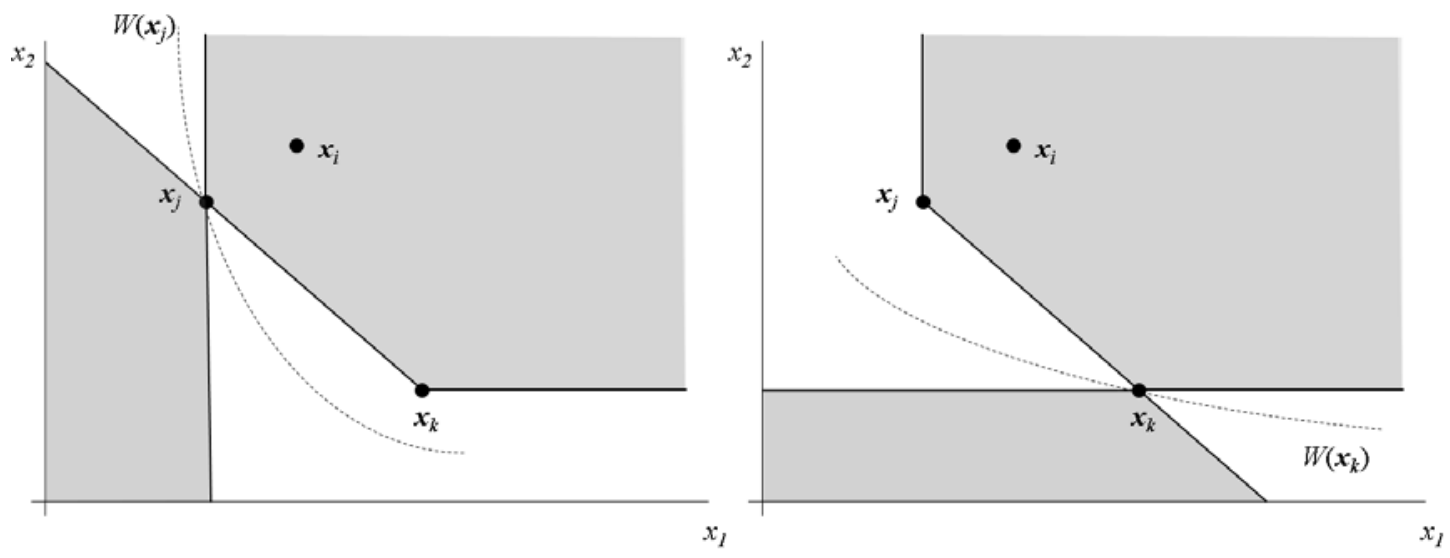
The last Figure (2C) combines the bounds in the cases that either $j$ or $k$ are the worst off observations. The shaded areas in Figure $2 \mathrm{C}$ represent the intersection of the corresponding areas in Figure 2B and are upper and lower bounds on all possible reference welfare curves which are consistent with monotonic and quasiconcave welfare functions and the restriction that they are referenced on the worst off observation. Since we now have bounds on the reference welfare curve we can immediately recover bounds on the distance function. Figure $2 \mathrm{C}$ also overlays these bounds. The distance measure for observation $i$ must be such that the deflated variable vector $d_{i} \mathbf{x}_{i}$ lies somewhere on the line between the points $\bar{D}_{i} \mathbf{x}_{i}$ and $\underline{D}_{i} \mathbf{x}_{i}$.

Figure 2C: Two -sided bounds on the distance function

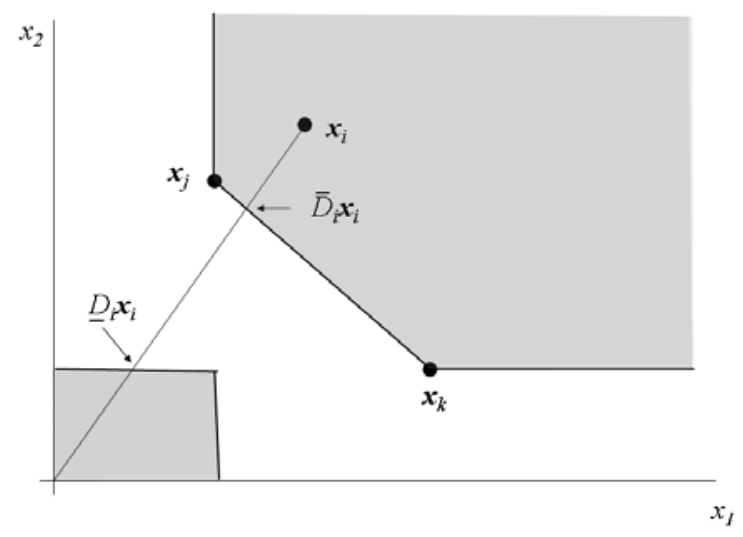

Using the result in Proposition 1 we can compute two-sided bounds on the distance index for each multivariate observation in a given dataset. It is worth noting in passing that these bounds are invariant to changes in units.

Proposition 2. Invariance to changes in units. If $d\left(\mathbf{x}_{i}, W^{*}\right) \in\left[\underline{D_{i}}, \overline{D_{i}}\right]$ then $d\left(\mathbf{k x}_{i}, W^{*}\right) \in$ $\left[\underline{D_{i}}, \overline{D_{i}}\right]$ where $\mathbf{k}$ is a $(m \times 1)$ vector of positive constants ${ }^{3}$.

\section{Proof. See appendix.}

To summarise; we are proposing a method which provides bounds on the set of all distance measure-based welfare indices which are consistent with the class of welfare aggregators which are monotonic and quasiconcave. These measures can be used to rank multivariate observations in a way which is invariant to choice of units. The use of the distance function as a tool with which to investigate welfare aggregation issues has been used before (for example, Lovell

\footnotetext{
${ }^{3}$ Note that $\mathbf{k x}_{i}$ denotes the element-by-element multiplication (Hadamard product) of the two vectors.
} 
et al (1994), Deutsch et al (2003) and Ramos and Silber (2005)) by authors who estimate the distance function using a translog specification linking a reference variable to the other dimensions. The problems with this general approach are well known and centre on the possibility of econometric misspecification conditional on the assumed functional form. In this paper we have put forward a nonparametric alternative similar in spirit to data envelopment and revealed preference approaches. The main difference from data envelopment analysis is that whilst in a standard envelopment problem both inputs and outputs are observed, we only observe inputs. We use restrictions on the class of admissible welfare functions (monotonicity and quasiconcavity) and a particular choice of reference observation (the worst off) to allow us to get around this problem. The main weakness of this type of approach is similar to the problems of standard envelopment problems: the results are data-dependent and so can sometimes be influenced by outliers and may consequently be determined by relatively few, extreme (low) observations.

\section{An Empirical Illustration: International Development}

We focus on a now well-established measure of international development produced by the United Nations, the Human Development Index (HDI). Data was taken from the UNDP (2009) Human Development Report 2009, which measures information for the year 2007 on 182 nations ${ }^{4}$. There are three indicators of well-being - life expectancy at birth in years, education (measured as a combination of indicators of adult literacy and the combined enrolment rate in all levels of education), and GDP per capita, measured in US dollars at purchasing power parity. The HDI is calculated by comparing the value of each indicator to benchmark upper and lower levels. This produces three indices between zero and one which represent the extent to which a country has moved towards the upper benchmark. For example, the life expectancy benchmarks are 25 years and 85 years. A country with a life expectancy of 25 years or lower would get an index of zero; a country with a life expectancy of 85 years or more receives an index of one. In 2007, the UK's life expectancy was 79.3 years which gave it a life expectancy index of $\frac{79.3-25}{85-25}=0.906$. The overall HDI is a simple average of the life expectancy, education and GDP indices. Our distance measures are calculated from the three component indices.

As discussed in the introduction, the HDI is an existing example of attempts to combine multiple indicators of well-being into a single index and highlights clearly many of the issues in doing so. Even assuming that the indicators are measured reliably and comparably across

\footnotetext{
${ }^{4}$ Source data is available from http://hdr.undp.org/en/media/HDR_2009_Tables.xls.
} 
countries, and each index is meaningful in itself (capping the maximum possible life expectancy at 85 means, for example, that a country with a life expectancy of 100 would be no more 'developed' than one with a life expectancy of $85^{5}$ ), assigning equal weight to each is clearly arbitrary. Analysis from the 2008 update of the HDI figures (UNDP, 2008) suggested that "... 70 percent of all possible country-pair comparisons are fully robust, meaning that the rankings would not be reversed at any non-negative weights that sum to 1." This, of course, reflects all possible pairwise comparisons and it is hard to imagine that countries at the top of the index would ever fall below those at the bottom on any re-weighting of the data. We may expect much more fluctuation in the ranks of countries close to one another. The report does point out that "... at some parts of the distribution, including among the top ten countries ... the rankings are sensitive to changes in the weights of the underlying components."

FiguRE 3. Welfare bounds

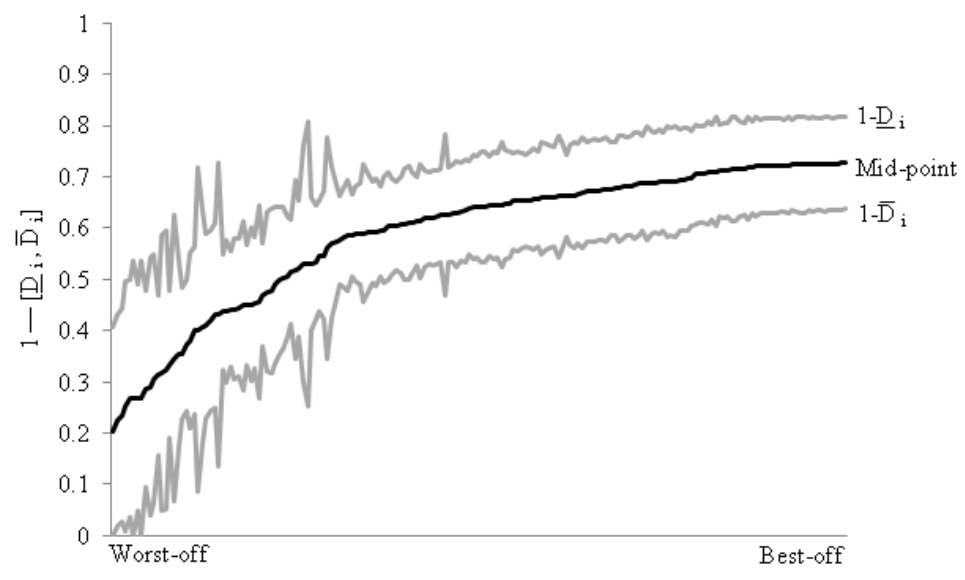

We took the data published in the Human Development Report 2009 and computed, for each country, bounds on the distance index as described in the previous section. Figure 3 shows the countries ordered from worst-off to best-off according to the mid-point of their distance bounds, along with the lower and upper bounds. Recall that higher values of the distance measure represent lower welfare. For ease of exposition, we have subtracted the bounds from 1 such that higher values reflect higher welfare. The results indicate that the bounds on the distance measure are informative about welfare comparisons across countries: the bounds do not span the entire interval $[0,1]$ and on average across all countries, the gap between upper

\footnotetext{
${ }^{5}$ For the income index, the upper limit is set at $\$ 40,000$ per capita at PPP. Thirteen of the 182 countries studied had incomes above this value meaning that Liechtenstein, with an income of $\$ 85,382$ per capita, has the same GDP index as Switzerland despite the latter's income per capita being half as much $(\$ 40,658$ per capita).
} 
and lower bound is 0.246 . Using the mid-point of the bound to rank countries, the best-off nation is Norway, which has an interval [0.637,0.818]. Of the 182 nations in the HDI, 38 have bounds that do not overlap the Norwegian bounds at all. Similarly, ranking by the mid-point the worst-off country is Niger, with bounds [0.000,0.407]. In total, 132 countries have bounds that do not overlap those of Niger.

In general, the size of the interval of the bounds is decreasing in overall welfare. Particularly noticeable is that the bounds are typically narrowest for better-off nations. Of the 68 countries with a mid-point below 0.6 , the average width of the bounds is 0.330 whilst the 114 countries with a mid-point in excess of 0.6 have an average width of 0.196 . The largest interval is Swaziland $([0.086,0.718])$ and the narrowest interval is Vietnam $([0.507,0.665])$. Conceptually, both the overall magnitude of the welfare inputs and their variability may be important determinants of the width of the bound. The correlation coefficient between the interval width and the mean of the life expectancy, education and GDP indices is -0.801 which mirrors the result from figure 3 that better-off nations have tighter bounds. The correlation coefficient between the interval width and the standard deviation of the indices is +0.401 , suggesting countries with more variable inputs tend to have wider bounds.

The Human Development Report classifies countries with an HDI in excess of 0.9 as "very high human development", countries with an HDI between 0.8 and 0.9 as "high human development", countries with an HDI between 0.5 and 0.8 as "medium human development" and countries with an HDI below 0.5 as "low human development". Figure 4 re-orders countries according to their HDI (worst-off on the left), demarcating these different development rankings, and shows the distance bounds as above.

FiguRE 4. Distance measures by HDI development classification

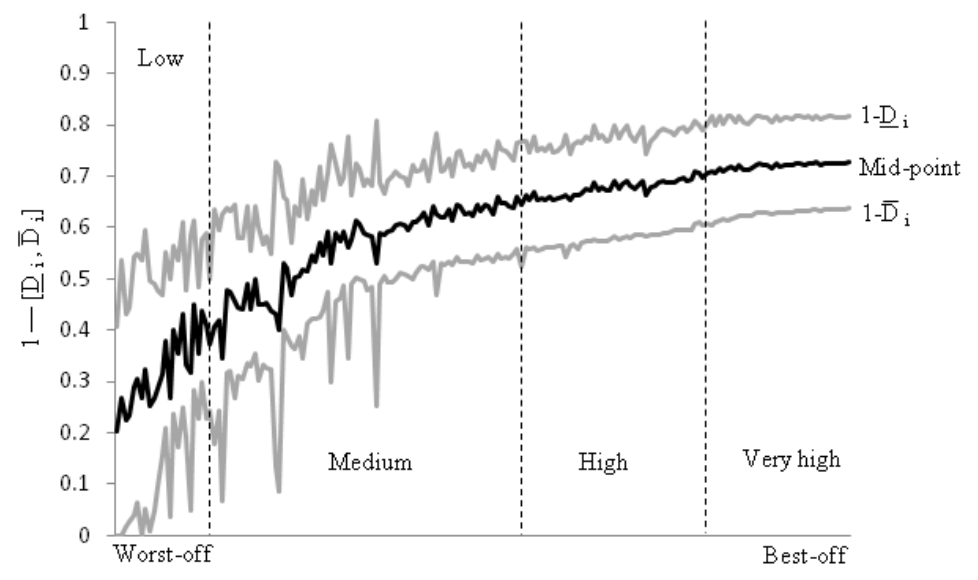


Clearly there is a close relationship between HDI and the distance measures suggesting the particular choice of aggregation method in the HDI is quite robust. The variation in the bounds for countries classed as 'very high development' is particularly small. However it is clear that there is some overlap between the groups. Taking the mid-point of the bounds as a welfare measure, 4 of the 38 countries ranked 'very high development' by the HDI have mid-points below that of Bahrain which is the best-ranked (by mid-point) of those countries grouped 'high development'. Similarly, 9 of the 24 nations classified as 'low' development have mid-points that would rank them higher than Lesotho, the 'medium' development country with the lowest mid-point. There is, however, no overlap of the mid-points across two development categories - no 'medium' development nation has a mid-point higher than that of any 'very high' development nation, and similarly for the 'low' and 'high' development groups.

Since the lower bound of $1-D_{i}$ (corresponding to the upper bound of $D_{i}$ ) is the lowest value a monotonic quasi-concave welfare index could take on for agent (country) " $i$ ", changes in these bounds across a group of agents are of interest in their own right since they are changes in the worst case scenario. Furthermore, those countries for whom $1-\mathrm{Di}=0$ are potentially the poorest in the population, what we have referred to elsewhere as the "Rawlsian Set" (Anderson et al (2006)), membership of this set over a span of time contradicts the "Rawlsian Improvement" criteria. Pooling data for the 172 countries for which data was available for years 1999 and 2008 yields the following distributions of upper and lower bounds for $1-D_{i}$.

Figure 5: Size Distributions of Upper and Lower Bounds

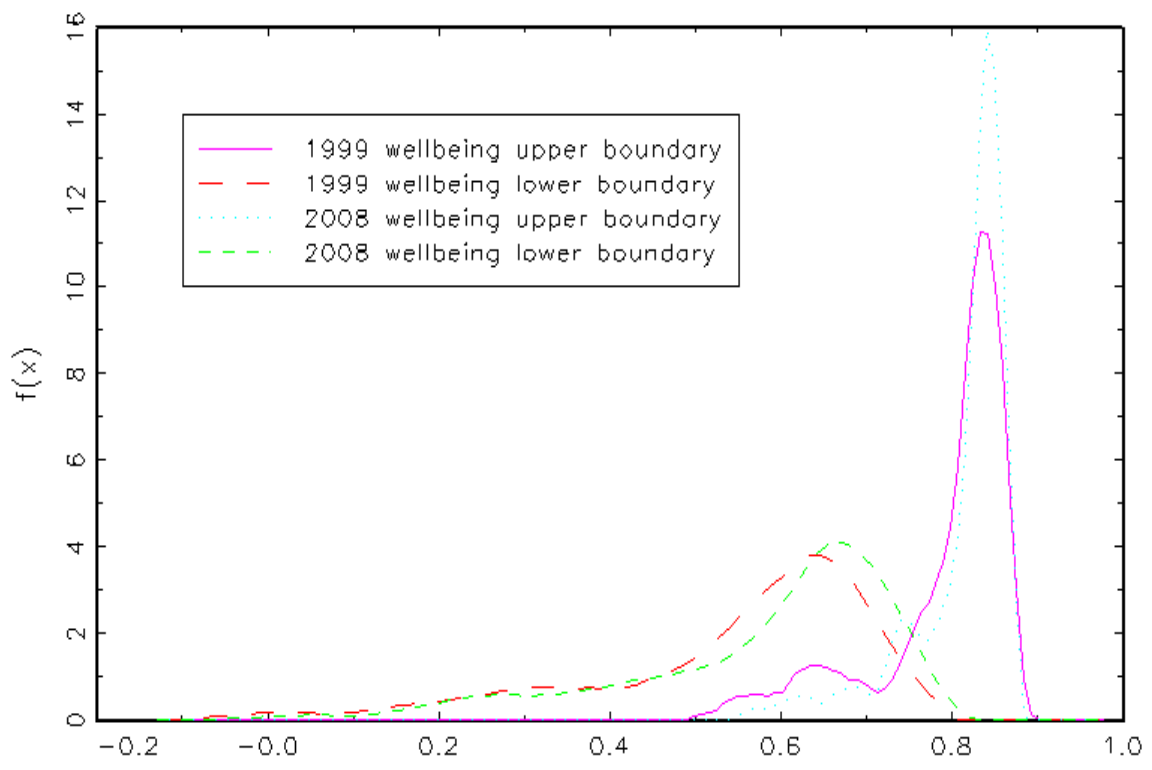


TABLE 1: Dominance results

\begin{tabular}{llllll}
\hline \hline Decile & & $F_{1999}$ & $F_{2008}$ & $\Delta F$ & std err \\
\hline 1 & 0.2982 & 0.1047 & 0.0930 & 0.0116 & 0.0322 \\
2 & 0.4414 & 0.2093 & 0.1860 & 0.0233 & 0.0429 \\
3 & 0.5274 & 0.3197 & 0.2791 & 0.0407 & 0.0493 \\
4 & 0.5820 & 0.4419 & 0.3546 & 0.0872 & 0.0526 \\
5 & 0.6197 & 0.5640 & 0.4360 & 0.1279 & 0.0535 \\
6 & 0.6387 & 0.6802 & 0.5174 & 0.1628 & 0.0521 \\
7 & 0.6654 & 0.7733 & 0.6221 & 0.1511 & 0.0488 \\
8 & 0.6881 & 0.8547 & 0.7442 & 0.1105 & 0.0428 \\
9 & 0.7069 & 0.9477 & 0.8488 & 0.0988 & 0.0322 \\
10 & 0.7257 & 1 & 1 & & \\
\hline \hline
\end{tabular}

Table 1reports the first order dominance test statistics (Davidson and Duclos (2000)) for the distribution of lower bounds which clearly fail to reject the hypothesis that the 2008 distribution first order dominates the 1999 distribution and rejects the hypothesis that the 1999 distribution dominates the 2008 distribution. The membership of the "Rawlsian Set" is four in number, Burkino Faso, Niger and Sierra Leone in 1999 and Democratic republic of the Congo in 2008 suggesting that that country actually went backwards over the period. Since the "Rawlsian Set" did not uniquely contain 1999 observations it is not possible to conclude that a Rawlsian welfare improvement has occurred over the period.

\section{Conclusions}

Measuring the relative social and economic welfare of individuals, households and countries is difficult, especially when welfare is characterized by a potentially large number of dimensions. Nevertheless, it is important to try to do so if we want to understand phenomena like social exclusion, poverty etc., and the effects of policy and environmental changes on them. The key problem is, perhaps, one of aggregation: we often have a fairly good idea of the sorts of factors which relate to overall welfare, but not how to aggregate these various dimensions into a single, useful index of relative welfare. This paper has offered a constructive approach to this problem. We show how two-sided bounds can be placed on a welfare indices using weak assumptions on the properties of the welfare function and a suitable choice of reference observation. Our approach is fully nonparametric and straightforward to apply to any multi-dimensional dataset as it only requires standard linear programming techniques. We illustrated the method using the UN HDI data and obtained informative two-sided bounds on the welfare index which could be used both to make welfare comparisons and also to check the robustness of the HDI methodology. The approach described in this paper also suggests a potential research 
program which might extend this method in two ways. The first is the investigation of the effects of further assumptions on the class of admissible welfare functions. Since we only use two rather weak assumptions there is considerable potential scope for tightening the bounds by adding further assumptions - two obvious candidates might be, for example, a separable or even additive structure for the welfare function. The second avenue for further research is to allow for the effects of sampling variation on the bounds. In this paper we assume that the constituent welfare indicators are measured correctly, but this may well not be the case and it would be important to extend the work here to consider the issue of statistical inference. Finally, it may be possible to use numerical values of the welfare inputs to further tighten the bounds or make comparative welfare comparisons across countries - this method does not rule out the possibility that an observation with high values of all inputs will have bounds that overlap with those of another observation with lower values of all inputs, but clearly monotonicity alone allows us to say the welfare of the former exceeds that of the latter. 


\section{Proofs}

\section{Proof of Proposition 1.}

Consider the upper bound. Let $\boldsymbol{\lambda}$ and $\overline{D_{i}}$ denote the solutions to the linear programme given in the definition of $\overline{D_{i}}$. Now suppose that $d\left(\mathbf{x}_{i}, W^{*}\right)>\overline{D_{i}}$. Since $\boldsymbol{\lambda} \geq \mathbf{0}_{n}, \mathbf{1}_{n}^{\prime} \boldsymbol{\lambda}=1$ and $\overline{D_{i}} \mathbf{x}_{i}=\mathbf{X} \boldsymbol{\lambda}$, we have $W\left(\overline{D_{i}} \mathbf{x}_{i}\right)=W(\mathbf{X} \boldsymbol{\lambda}) \geq W^{*}=\min _{j}\left\{W\left(\mathbf{x}_{j}\right): \mathbf{x}_{j} \in \mathbf{X}\right\}$ for all aggregators satisfying $\mathrm{A} \rightarrow$ and $\mathrm{A} 2$. Therefore, if $d\left(\mathbf{x}_{i}, W^{*}\right)>\overline{D_{i}}$, then $W\left(d\left(\mathbf{x}_{i}, W^{*}\right) \mathbf{x}_{i}\right)>$ $W\left(\overline{D_{i}} \mathbf{x}_{i}\right)$ which implies that $d\left(\mathbf{x}_{i}, W^{*}\right)>\min _{d \geq 0}\left\{d: W\left(d \mathbf{x}_{i}\right) \geq W^{*}\right\}$ which contradicts the definition of the distance function. Now consider the lower bound and suppose that $\underline{D_{i}}>d\left(\mathbf{x}_{i}, W^{*}\right)$. This implies that $\min _{m}\left\{d\left(\mathbf{x}_{i}, W^{*}\right) x_{i}^{m}\right\}<\min _{m}\left\{\min _{j}\left\{x_{j}^{m}\right\}\right.$ for $\left.j=1, \ldots, n\right\}$ where

$\min _{m}\left\{\min _{j}\left\{x_{j}^{m}\right\}\right.$ for $\left.j=1, \ldots, n\right\}=W^{*}$ for the Leontief aggregator function (which satisfies A1 and A2) and hence that $W\left(d\left(\mathbf{x}_{i}, W^{*}\right) \mathbf{x}_{i}\right)<W^{*}$ which contradicts the definition of the distance function.

\section{Proof of Proposition 2.}

Consider the lower bound and note that $x_{j}^{m} / x_{i}^{m}=k^{m} x_{j}^{m} / k^{m} x_{i}^{m}$ so invariance follows immediately. Now consider the upper bound and suppose that $\overline{D_{i}}$ solves the linear program described in Theorem 1. Then $\overline{D_{i}} \mathbf{x}_{i}=\mathbf{X} \boldsymbol{\lambda}$ and hence $\overline{D_{i}}$ also satisfies $\overline{D_{i}}\left[\mathbf{k} \mathbf{x}_{i}\right]=[\mathbf{k X}] \boldsymbol{\lambda}$. 


\section{References}

[1] Anand, S. and A. K. Sen (1997), "Concepts of human development and poverty: a multidimensional perspective", Human Development Papers, New York: UNDP

[2] Anderson, G.J., Crawford I. and A. Leicester "Efficiency Analysis and the Lower Convex Hull Approach" Chapter 10 in Quantitative Approaches to Multidimensional Poverty Measurement. Kakwani N., and J. Silber eds. Palgrave McMillan 2008.

[3] Atkinson, A. B. (2003), "Multidimensional deprivation: contrasting social welfare and counting approaches", Journal of Economic Inequality, vol 1 (1), 51-65

[4] Bourguignon, F. amd S. R. Chakravarty (2003), "The measurement of multidimensional poverty", Journal of Economic Inequality, vol 1 (1), 25-49

[5] Davidson, R and J-Y Duclos (2000) "Statistical Inference for Stochastic Dominance and for the Measurement of Poverty and Inequality. Econometrica 68, 2000, 1435-1464

[6] Deaton, A. (1979), "The distance function in consumer behaviour with an application to index numbers and optimal taxation", The Review of Economic Studies, vol 46 (3), 391-405

[7] Deaton, A. and J. Muellbauer (1980), Economics and Consumer Behavior, Cambridge: Cambridge University Press

[8] Deutsch, J., X. Ramos, and J. Silber, (2003), "Poverty and Inequality of Standard of Living and Quality of Life in Great Britain," in J. Sirgy, D. Rahtz, and A. C. Samli (eds), Advances in Quality-of-Life Theory and Research, Kluwer Academic Publishers, Dordrecht, $99-128$

[9] Grusky, D. B and R. Kanbur (2006), Poverty and Inequality: Studies in Social Inequality, Stanford: Stanford University Press

[10] Kolm, S. C. (1977), "Multidimensional egalitarianisms", Quarterly Journal of Economics, vol 91 (1), 1-13

[11] Lovell, C. A. K., S. Richardson, P. Travers, and L. Wood, (1994), "Resources and Functionings: A New View of Inequality in Australia," in W. Eichhorn (ed.), Models and Measurement of Welfare and Inequality, Springer-Verlag, Heidelberg 
[12] Maasoumi, E. (1986), "The measurement and decomposition of multidimensional inequality", Econometrica, vol 54 (4), 771-779

[13] Malmquist, S. (1953), "Index numbers and indifference surfaces", Trabajos de Estadistica, 4, 209-242.

[14] Ramos, X. and J Silber, (2005) "On the application of efficiency analysis to the study of the dimensions of human development", Review of Income and Wealth, 51 (2), 285-309

[15] Rockafellar, R. T. (1970), Convex Analysis, Princeton: Princeton University Press

[16] Sen, A. K. (1995), Inequality Reexamined, Harvard: Harvard University Press

[17] Shephard, R.W. (1953), Cost and Production Functions, Princeton: Princeton University Press

[18] United Nations Development Programme (2008), Human Development Indices: A statistical update 2008, New York: UNDP

[19] United Nations Development Programme (2009), Human Development Report 2009 Overcoming barriers: Human mobility and development, New York: UNHDP 